\title{
Weighted Proportional Fairness and Pricing Based Resource Allocation for Uplink Offloading Using IP Flow Mobility
}

\author{
V. Miliotis ${ }^{a, *}$, L. Alonso ${ }^{a}$, C. Verikoukis ${ }^{b}$ \\ ${ }^{a}$ Department of Signal Theory and Communications, Technical University of Catalonia \\ (UPC), Barcelona, Spain \\ ${ }^{b}$ Telecommunications Technological Centre of Catalonia (CTTC), Castelldefels, Spain
}

\begin{abstract}
Mobile data offloading has been proposed as a solution for the network congestion problem that is continuously aggravating due to the increase in mobile data demand. However, the majority of the state-of-the-art is focused on the downlink offloading, while the change of mobile user habits, like mobile content creation and uploading, makes uplink offloading a rising issue. In this work we focus on the uplink offloading using IP Flow Mobility (IFOM). IFOM allows a LTE mobile User Equipment (UE) to maintain two concurrent data streams, one through LTE and the other through WiFi access technology, that presents uplink limitations due to the inherent fairness design of IEEE 802.11 DCF by employing the CSMA/CA scheme with a binary exponential backoff algorithm. In this paper, we propose a weighted proportionally fair bandwidth allocation algorithm for the data volume that is being offloaded through $\mathrm{WiFi}$, in conjunction with a pricing-based rate allocation for the rest of the data volume needs of the UEs that are transmitted through the LTE uplink. We aim to improve the energy efficiency of the UEs and to increase the offloaded data volume under the concurrent use of access technologies that IFOM allows. In the weighted proportionally fair $\mathrm{WiFi}$ bandwidth allocation, we consider both the different upload data needs of the UEs, along with their LTE spectrum efficiency and propose an access mechanism that improves the use of WiFi access in uplink offloading. In the
\end{abstract}

\footnotetext{
${ }^{*}$ Corresponding author (Vasileios Miliotis). Tel. +34 934137147. E-mail address: vasileios.miliotis@tsc.upc.edu.
} 
LTE part, we propose a two-stage pricing-based rate allocation under both linear and exponential pricing approaches, aiming to satisfy all offloading UEs regarding their LTE uplink access. We theoretically analyse the proposed algorithms and evaluate their performance through simulations. We compare their performance with the 802.11 DCF access scheme and with a state-of-the-art access algorithm under different number of offloading UEs and for both linear and exponential pricing-based rate allocation for the LTE uplink. Through the evaluation of energy efficiency, offloading capabilities and throughput performance, we provide an improved uplink access scheme for UEs that operate with IFOM for uplink offloading.

Keywords: Offloading, energy efficiency, uplink, IFOM, fairness, pricing

\section{Introduction}

The continuous increase of cellular data demand that is already witnessed, is the main driving force for cellular network operators towards the capital investments on upgrades of their cellular network infrastructures into 4G systems, as LTE. With the upgrade of their networks, cellular providers aim to be able to serve the requested traffic by their customers. Despite the upgrade of the cellular infrastructures, the pace of the increase of the data traffic demand [1] puts pressure on the cellular network providers, as traffic congestion is not avoided. These facts have led the research community to propose offloading techniques that will leverage the mitigation of the overload of the cellular network spectrum and the network's traffic congestion.

According to the work of Paul et al. [2] on the dynamics of cellular data networks, downloads dominate uploads with more than $75 \%$ of the traffic coming from download traffic. On the other hand, smartphone applications slowly change the users attitude, transforming them into content creators. Facebook, Twitter, Youtube and Instagram are some of the main applications that let users upload their content (videos, photos, audio, text and combinations of them) at the time of creation. This change of use habits is highly demanding in terms of energy consumption, as in LTE, uploading is nearly eight times more energy consuming compared to downloading according to the extensive measurements of [3]. In the same work it is experimentally measured that LTE consumes two times the energy of WiFi for uploading small files of size equal to $10 \mathrm{kB}$ and 2.53 times the energy of WiFi for larger files of size equal to $10 \mathrm{MB}$. Considering the solution of offloading the uplink 
traffic of users that are in the range of WiFi Access Points (APs), the battery life of mobile users will be extended and at the same time the uplink load of an eNodeB will be mitigated. According to Cisco's mobile data traffic forecast [1], mobile offload increases from $45 \%$ (1.2 exabytes/month) that was in 2013 to $52 \%$ (17.3 exabytes/month) by 2018. As operators will not likely be able to keep pace with the current pace of mobile data demand, they respond by rolling out WiFi APs to public areas to offload data traffic. WiFi is an appropriate solution, as WiFi APs are easier to deploy and they cost less than upgrading existing cellular infrastructure gear.

With the release-10 of 3GPP, a UE in LTE networks is able to concurrently maintain connections with the cellular network and a WiFi AP, in order to offload part of its traffic through WiFi access and upload the rest through LTE. The scheme that allows this connectivity is named IP Flow Mobility (IFOM) [4]. The other two offloading techniques are Local IP Access (LIPA) and Selected IP Traffic Offload (SIPTO). IP Flow Mobility is currently being standardized by 3GPP [5]. This technology allows an operator or a UE to shift an IP flow to a different radio access technology, without disrupting any ongoing communication. Consider a UE connected to a cellular base station having multiple simultaneous flows. For example, it maintains a voice call and a file upload, and it is moving into the range of a WiFi AP. The UE may shift the file upload on the WiFi network and when it moves out of the AP coverage it will make a seamless shift of the flow back to the cellular network. Another example is the division of a UE's data flow into two sub-flows and the service of each sub-flow by different radio access technologies, as proposed in [6]. In [7], the authors introduce the first peer-assisted method for offloading traffic from the uplink, and the selection of the most cost-efficient alternative access technology for data transmission.

A question that arises from the IFOM uplink offloading scheme is how the UEs will offload part of their data through WiFi with fairness, where their different upload data needs and their LTE connection with the eNodeB will be considered, and how the rest of the data will be uploaded through LTE. Although the access method in 802.11 DCF (Distributed Coordinated Function), uses the CSMA/CA protocol to share radio resources in a fair way, it treats all users equally. This access scheme creates unfairness considering the different data needs of each UE and the different channel conditions of their connection with the eNodeB. In cases where different queue lengths are considered [8] or in multi-rate conditions [9], fair resource allocation is achieved by weighted proportional fairness. While the downlink of a WiFi 
AP can be adaptive, based on priority queuing of data, e.g. by applying the 802.11e standard [10], the uplink does not present the same flexibility. In uplink, all transmitting users are treated equally, following the binary exponential backoff algorithm of 802.11 DCF. Based on this fact we focus on providing an effective access scheme for uplink offloading through WiFi that will treat all UEs on a weighted proportionally fair way, which includes the UEs uplink data needs in conjunction with their LTE channel conditions. The main objective of this approach is to achieve energy efficiency and throughput improvement in the uplink offloading with IFOM.

The main challenge of our work is to provide an efficient uplink offloading algorithm that takes into consideration the different uplink data volume needs of UEs that are associated with the same WiFi AP and eNodeB, and present different channel conditions regarding their LTE uplink. The main questions that are tackled throughout this paper are the following: (i) How the different data needs of UEs under the coverage of the same eNodeB and WiFi AP should be divided into two sub-flows per UE that will be concurrently routed through the available access technologies? (ii) How can we improve the WiFi uplink access to maximize the uplink offloading of the data volume needs of the UEs? and (iii) How can we provide an efficient resource allocation for the LTE uplink of the data volume needs of the UEs that are not offloaded through WiFi? In this paper we discuss on the limitations of IEEE 802.11 DCF uplink access and we propose an offloading algorithm for IFOM that combines weighted proportional fairness in the WiFi access and price-based resource allocation in the LTE upload. UEs that have larger upload data needs or experience worse LTE connection are favoured in the WiFi offloading part. This is achieved by choosing appropriate weights for the proportional fairness. The LTE uplink rate allocation we propose is a two-stage pricing algorithm. In the first stage, the LTE operator decides the price $p$ per unit of a UE's LTE uplink rate. In the second stage, the UEs decide the rate for which they intend to pay, based on the price and the spectrum efficiency that they experience. Data pricing has been recently adopted as a promising economics tool that provides effective solutions for resource allocation aiming to mitigate network congestion [11]. We follow two different pricing schemes. A linear pricing scheme, that was used in [12] and [13] and an exponential pricing scheme, that was used in [14]. The main contributions of this work are the following:

- To the best of our knowledge this is the first work that considers uplink 
offloading methods for WiFi and LTE networks that operate under the IFOM offloading technique.

- We propose a weighted Proportionally Fair Bandwidth (PFB) allocation algorithm for the $\mathrm{WiFi}$, aiming to improve the uplink offloading. We include in the fairness criteria the different data needs of the UEs and their LTE uplink spectrum efficiency.

- For the rest of each UE's data we propose a price-based rate allocation for the LTE uplink, and we follow a linear and an exponential pricing scheme. Our major focus is to investigate the effect of different pricing schemes on the energy efficiency and throughput performance of UEs under IFOM uplink offloading.

We compare the PFB algorithm with 802.11 DCF and with a state of the art uplink access scheme in terms of UEs' energy efficiency for both linear and exponential pricing of the LTE rate allocation. We investigate the conditions under which exponential pricing performs better than linear pricing and we reveal the effect of the UEs' data needs and spectrum efficiency on their energy efficiency and throughput performance. In addition, we evaluate the offloading capabilities of PFB and we show that a greater data volume is offloaded using our proposed algorithm.

\section{Related work}

The relatively low deployment costs of WiFi APs has led the providers and the research community to investigate offloading techniques for the cellular networks through WiFi. In [15], the authors have indicated that WiFi already offloads in US about $65 \%$ of the total mobile data traffic and saves $55 \%$ of battery power. In the same work, the offloading capabilities of WiFi were investigated under trace-driven simulations, based on mobility habits of mobile users and useful insights have been provided on temporal offloading. In [16], offloading through opportunistic communications was explored, where a user offloads to another peer user, which in its turn maintains a short range connection (e.g. WiFi or Bluetooth) or a cellular connection (e.g. EDGE or HSPA). The problem of device-to-device communications over locally formed ad hoc networks has been also addressed in [17], in the context of the download process. According to their model, the base station transmits different parts of the content to selected mobile devices. Following, the 
mobile devices multicast the received data to each other. The combination of device-to-device communication with delay-tolerant traffic was proposed in RoCNet [18], where a user terminal under the coverage of a high traffic loaded BS, forwards its traffic through a WLAN or a Bluetooth connection to another user terminal, which will be physically moved under the coverage of another cellular BS with low traffic load to offload its peers uplink data. In [19], the authors have proposed Wiffler, which is an application that is used to predict WiFi connectivity aiming to leverage the exploitation of offloading opportunities. Through the conducted measurements in city-wide testbeds they found that cellular and WiFi availability are negatively correlated, a fact that expands the benefit of offloading through WiFi in terms of network coverage. The authors in [20] have provided a study on the economics of mobile data offloading through third-party WiFi or femtocell APs and they proposed a market-based offloading scenario, aiming to investigate the market outcome with game theory. In [21], the authors proposed a framework named iDeal, that allows providers to use resources from third-party resource owners, by leasing capacity in cases of congestion through reverse auctions. Several third-party resource owners were considered, that compete to lease their resources to the cellular provider, leading to significant savings for the provider's side. An optimal delayed WiFi offloading algorithm was proposed in [22]. The authors considered the case of file downloading by mobile users that move under the BreadCrumbs mobility model, proposed in [23], and they provided an optimal algorithm that minimizes the mobile user's communication cost. In [24], methods for session continuity were proposed for non-seamless WiFi offloading in LTE networks. The performance of these methods was analysed in terms of throughput and energy consumption. In [25], the authors analysed the behaviour of the network decision-making and reconfiguration process in terms of handled handover requests, aiming to achieve load balancing by guiding the relocation of mobile terminals to achieve offloading. An enhanced framework for Wi-Fi-based offloading was proposed in [26], where the author investigated how to raise Quality of Experience (QoE) by evaluating the deadline assurance in offloading, while saving a significant amount of $3 \mathrm{G}$ resources. In [27], the authors considered a model where a subset of users under the coverage of a base station are at the same time under WiFi coverage, and they aimed to maximize the per-user-throughput by selecting which WiFi connected users would be allowed to offload, taking into consideration the induced collisions for nonsaturated traffic. The energy efficiency under this offloading scheme is left 
open. The recent published works related to offloading are mainly focused on the downlink traffic offloading and did not consider the increasing tendency of uploading user created content [28]. In our work we raise awareness of the uplink traffic offloading and its impact on the energy efficiency of the modern mobile communication devices. Contrary to [27] we consider that all UEs under the concurrent coverage of an eNodeB and a WiFi AP are given the opportunity to offload, following a weighted fair allocation for WiFi and a pricing scheme for LTE, while transmitting concurrently through the aforementioned access technologies. In [29], we presented the uplink offloading system model based on IFOM, and we proposed a heuristic solution along with a simple proportional fair approach weighted only by the data needs of the UEs. In [30], we provided a mathematical proof for the simple weighted proportionally fair WiFi bandwidth allocation. In this paper an enhanced proportionally fair solution is provided with a mathematical proof, and in conjunction with linear and exponential pricing algorithms, for the resource allocation regarding the LTE uplink access.

The rest of the paper is organised as follows. In Section 3 we present the system model and we analyse the energy consumption of the LTE and WiFi network interface cards of UEs. In Section 4 we analyse the PFB algorithm and in Section 5 we analyse the proposed LTE pricing scheme for linear and exponential pricing. Section 6 contains the evaluation and simulation results and Section 7 concludes our work.

\section{System Model}

The main notations used in this paper are summarized and explained in Table 1 for the ease of reading. We consider a LTE macro-cell and we focus on its coverage area that is also partially covered by several WiFi APs that belong to the same LTE provider, as shown in Fig. 1. All UEs are equipped with a WiFi network interface card in addition to their LTE connectivity. We assume that $N$ LTE UEs are concurrently under the coverage of the macro-cell and one of the deployed APs, and they need to upload a file (e.g. a photo or a video) through a mobile application. The used applications are assumed to be able to divide an IP flow into two sub-flows and to define the size of each one. The UEs are able to use concurrently the two access technologies with IFOM and direct one sub-flow to LTE and the other to WiFi. The data volume $\mathrm{UE}_{i}$ needs to upload is equal to $K_{i}$ bits, where $i=(1, \ldots, N)$. The data needs $K_{i}$ are assumed a priori known to the WiFi 
Tab. 1. List of notations and their physical meanings.

\begin{tabular}{ll}
\hline \hline Symbols & PhysicalMeanings \\
\hline $\mathrm{UE}_{i}$ & UEs under the coverage of the same eNodeB and WiFi AP, $i=(1, \ldots, N)$ \\
$K_{i}$ & Uplink data volume needs of $\mathrm{UE}_{i}$ \\
$\Delta T$ & Duration of an uplink offloading period \\
$\theta_{i}$ & Normalized spectrum efficiency of $\mathrm{UE}_{i}, \theta_{i} \in[0,1]$ \\
$P_{i}^{L T E}$ & Power level of $\mathrm{UE}_{i}$ 's LTE interface during uplink transmission \\
$R_{i}^{L T E}$ & LTE uplink rate of UE (in Mbps) \\
$R_{\text {max }}^{L T E}$ & Maximum value of LTE uplink rate (in Mbps) \\
$\alpha_{u}$ & Uplink transmission power per Mbps \\
$\beta$ & Base power of the LTE card \\
$S(N)$ & Per UE WiFi uplink throughput (in Mbps), for $N$ contending UEs \\
$E E(N)$ & Per UE WiFi energy efficiency (in bits/Joule), for $N$ contending UEs \\
$R^{W i F i}$ & WiFi data transmission rate \\
$w_{i}$ & Offloading factor of UE,$w_{i} \in[0,1]$ \\
$E C_{T x}^{W i F i}$ & Average per UE energy consumption during WiFi transmission (Joule) \\
$E C_{\text {sleep }}^{W i F i}$ & Average per UE energy consumption during WiFi sleep phase (Joule) \\
$E C^{L T E}$ & Average per UE energy consumption during LTE transmission (Joule) \\
$E_{\text {eff }}^{P F B}$ & Average per UE energy efficiency of IFOM uplink offloading (bits/Joule) \\
$O f f_{P F B}$ & Offloading index of the PFB algorith \\
$R_{i}^{L T E}$ & LTE uplink transmission rate of UE \\
$p$ & Price per unit of LTE uplink transmit rate for linear pricing \\
$p_{e}$ & Price per unit of LTE uplink transmit rate for exponential pricing \\
$U_{i}^{\text {in }}$ & Payoff function of UE, for linear pricing \\
$U_{i}^{e x p}$ & Payoff function of UE, for exponential pricing \\
\hline \hline
\end{tabular}

AP. The AP has a high bandwidth backbone (e.g. fiber connection). Thus, the bottleneck of this route lies in the wireless uplink access of the WiFi connection. The described scheme is applied to each one of the WiFi APs 


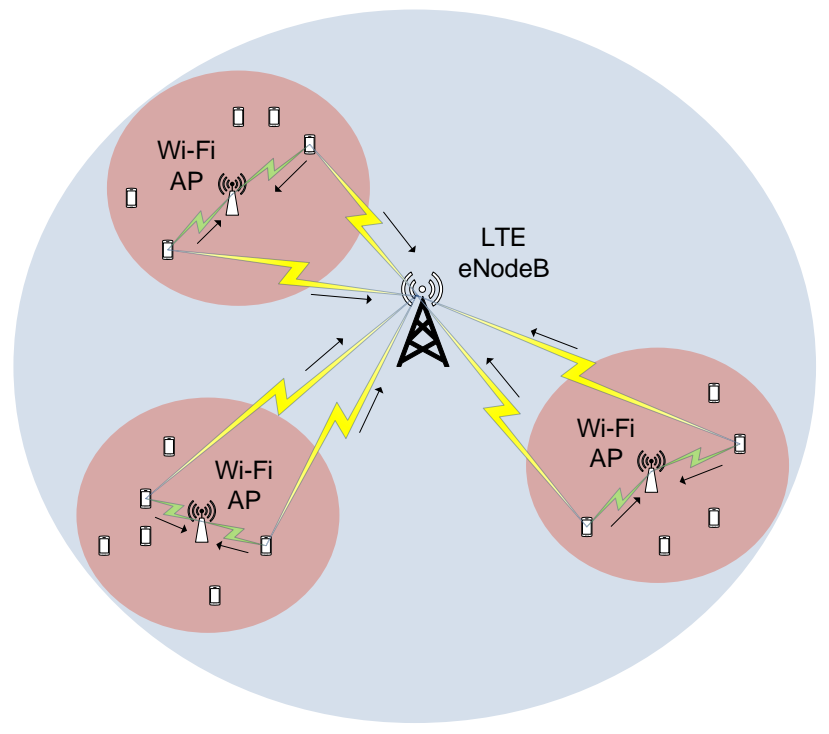

Fig. 1. Uplink offloading scenario with IP Flow Mobility (IFOM).

and we investigate the uplink data offloading for a time duration equal to $\Delta T$. Each $\mathrm{UE}_{i}$ offloads part of each data needs $K_{i}$. The rest is uploaded through its LTE connection. We assume that the channel characteristics between each $\mathrm{UE}_{i}$ and the LTE macro-cell are described by a normalized spectrum efficiency $\theta_{i} \in[0,1]$, such that for a bandwidth allocation that gives to $\mathrm{UE}_{i}$ the ability to upload with an uplink rate equal to $R_{i}^{L T E}$ under ideal channel conditions, the actual achieved uplink rate is equal to $\theta_{i} R_{i}^{L T E}$. As we focus on the access layer of the heterogeneous network, we assume that $\theta_{i}$ abstracts the physical layer characteristics including the frequency selectivity that the UEs may experience due to transmitting in different frequencies even with the same bandwidth. With this abstraction we provide a plug-in parameter to our access layer study, available to be used over a physical layer analysis. In Fig. 2 we provide a schematic representation of our proposed model, where we present which decisions are centralised at the operators side, and which are distributed at the UEs side. The operator decides how the weighted fair bandwidth allocation will be held at the WiFi offloading. The WiFi AP uses an exclusive access scheme to implement the bandwidth allocation decided by the operator, as presented in Section IV.A. Regarding the LTE rate allocation, the operator decides centrally the price $p$ per unit of LTE 


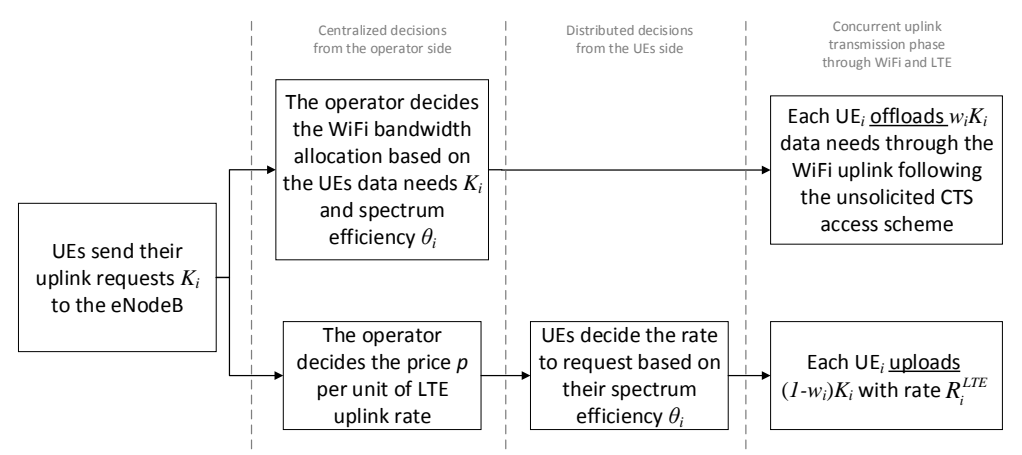

Fig. 2. Schematic representation of the system model.

uplink rate and following, the UEs distributively decide the LTE rate to request, based on the already decided price $p$ and their spectrum efficiency $\theta_{i}$. After these decisions, each $\mathrm{UE}_{i}$ transmits its data by concurrently using both access technologies.

\subsection{LTE Uplink Power Model}

Regarding the LTE uplink power level of the UE, we adopt the energy model proposed by Huang et al. in [3]. According to this model the power level of the $\mathrm{UE}_{i}$ 's LTE interface during uplink transmission is expressed as

$$
P_{i}^{L T E}=\alpha_{u} R_{i}^{L T E}+\beta[\mathrm{mW}]
$$

where $\alpha_{u}$ is the uplink transmission power per Mbps, $R_{i}^{L T E}$ is the LTE uplink rate (in Mbps) and $\beta$ is the base power of the LTE card.

\subsection{IEEE 802.11 DCF Energy Consumption in the Uplink}

The uplink access mechanism of IEEE 802.11 DCF [31] is based on contention among users that are willing to transmit data to the AP and try to avoid collisions following the standard's binary exponential back-off algorithm. Following Bianchi's analysis [32] for saturated traffic conditions we notice that the throughput of a user that tries to upload data through WiFi is significantly affected by the number of users that are under the coverage of the same AP. The per user uplink throughput $S(N)$ (in Mbps), where $N$ is the number of contending users, is expressed as 


$$
S(N)=\frac{P_{s}(N) P_{t r}(N) E[P]}{N\left[\left(1-P_{t r}(N)\right) \sigma+P_{t r}(N) P_{s}(N) T_{s}+P_{t r}(N)\left(1-P_{s}(N) T_{c}\right)\right]}
$$

$E[P], T_{s}, T_{c}$ and $\sigma$ correspond to the average payload of a packet, the duration of a successful transmission, the duration of a collision and the time slot's duration respectively. $P_{t r}(N)$ is the probability that there is at least one transmission in a considered time slot and $P_{s}(N)$ is the probability that an occurring transmission is successful. A user's energy efficiency $E E(N)$ (in bits/Joule), as a function of the number of contending users $N$ is expressed as

$$
E E(N)=\frac{P_{s}(N) P_{t r}(N) E[P]}{N\left[\left(1-P_{t r}(N)\right) E_{i}+P_{t r}(N) P_{s}(N) E_{s}+P_{t r}(N)\left(1-P_{s}(N) E_{c}\right)\right]}
$$

where $E_{i}, E_{s}$ and $E_{c}$ correspond to the energy consumption of a user during an idle, a successful transmission and a collision period. The duration of a successful transmission is equal to $T_{s}=T_{H}+T_{P}+T_{S I F S}+T_{A C K}+T_{D I F S}$. The duration of a collision period is equal to $T_{c}=T_{H}+T_{P}+T_{D I F S}$, and the duration of an idle period is equal to a time slot $\sigma$. Where $T_{H}$ is the transmission duration of the PHY and MAC headers and $T_{P}$ the transmission duration of a packet's payload for transmission rate equal to $R^{W i F i}=54$ Mbps. Taking these duration expressions into consideration we analytically express the energy consumption values of (3) in (4).

$$
\begin{aligned}
E_{s} & =P_{T x}\left(T_{H}+T_{P}\right)+P_{i d l e}\left(T_{S I F S}+T_{D I F S}\right)+P_{R x} T_{A C K} \\
E_{c} & =P_{T x}\left(T_{H}+T_{P}\right)+P_{i d l e} T_{D I F S} \\
E_{i} & =\sigma P_{i d l e}
\end{aligned}
$$

where $P_{i d l e}, P_{T x}$ and $P_{R x}$ are the power levels of the user's 802.11 network interface card.

\subsection{Uplink Offloading Energy Consumption}

Every UE under the concurrent coverage of the two access technologies will have the opportunity to offload $w_{i} K_{i}$ bits through the WiFi AP, where $w_{i} \in[0,1]$ for $i=(1, \ldots, N)$. The remainder data volume $\left(1-w_{i}\right) K_{i}$ is 
transmitted through the LTE connection of each UE. Every $\mathrm{UE}_{i}$ with data needs equal to $K_{i}$ that offloads its uplink according to $w_{i}$ will present energy consumption $E C_{i}(N)$, which is expressed as

$$
E C_{i}(N)=\left(1-w_{i}\right) K_{i} \frac{P_{i}^{L T E}}{\theta_{i} R_{i}^{L T E}}+w_{i} K_{i} \frac{1}{E E(N)} \text { [Joule] }
$$

For the WiFi uplink offloading we provide a weighted proportionally fair allocation algorithm over the data needs and the LTE channel conditions of the UEs. For the LTE uplink of the $\left(1-w_{i}\right) K_{i}$ data volume of each $\mathrm{UE}_{i}$ we provide a two stage pricing algorithm for the LTE uplink rate allocation. Based on these two parts of the IFOM uplink offloading we are able to calculate each $\mathrm{UE}_{i}$ 's energy consumption according to (5). Considering that without offloading a $\mathrm{UE}_{i}$ would upload with throughput equal to $\theta_{i} R_{i}^{L T E}$, we express the equivalent throughput of $\mathrm{UE}_{i}$ with offloading as $\theta_{i} R_{i}^{L T E} /\left(1-w_{i}\right)$, assuming that the LTE uploading continues after the WiFi offloading.

\section{Weighted Proportionally Fair WiFi Access}

The UEs offload part of their data needs through the WiFi, according to the Proportionally Fair Bandwidth (PFB) allocation algorithm that we propose. Each $\mathrm{UE}_{i}$ is allocated bandwidth equal to $r_{i}, i=(1, \ldots, N)$, such as $\sum_{i=1}^{N} r_{i} \leq R_{i}^{W i F i}$. The allocation is proportionally fair over the ratio $\rho_{i}=K_{i} / \theta_{i}$. According to the definition of proportional fairness by Kelly et al. [33], a vector of rate allocation $\mathbf{r}=\left(r_{1}, \ldots, r_{N}\right)$ is proportionally fair if it is feasible,

that is $\mathbf{r} \geq 0$ and $\sum_{i=1}^{N} r_{i} \leq R_{i}^{W i F i}$ and if for any other feasible vector $\mathbf{r}^{*}$, regarding the proportional fairness over the ratio $\rho_{i}$ of each $\mathrm{UE}_{i}$, the aggregate of proportional changes is zero or negative and is expressed as

$$
\sum_{i=1}^{N} \rho_{i} \frac{r_{i}^{*}-r_{i}}{r_{i}} \leq 0
$$

which can be rewritten as

$$
\sum_{i=1}^{N} \rho_{i}\left(\log \left(r_{i}\right)\right)^{\prime} d r_{i} \leq 0
$$


It follows from (7) that the proportionally fair allocation solution represents a maximum of the utility function $U_{i}(\mathbf{r})=\sum_{i=1}^{N} \rho_{i} \log \left(r_{i}\right)$. Consequently, in order to find the proportionally fair solution we have to solve the maximization problem described as follows

$$
\begin{aligned}
\max _{\mathbf{r}} & \sum_{i=1}^{N} \rho_{i} \log \left(r_{i}\right) \\
\text { subject to } & \sum_{i=1}^{N} r_{i} \leqslant R^{W i F i} \\
\text { and } & r_{i} \geqslant 0, \forall i=1, \ldots, N
\end{aligned}
$$

The problem has a unique solution since the objective function is strictly concave and the constraint set is convex. To solve this problem, we relax the constraints and define the Lagrangian [34], changing $r_{i} \geqslant 0$ to $-r_{i} \leqslant 0$

$$
L(\mathbf{r}, \mu)=\sum_{i=1}^{N} \rho_{i}\left(\log \left(r_{i}\right)\right)-\mu_{0}\left(\sum_{i=1}^{N} r_{i}-R^{W i F i}\right)+\sum_{i=1}^{N} \mu_{i} r_{i}
$$

where $\mu_{0} \geqslant 0$ and $\mu_{i} \geqslant 0, i=1, \ldots, N$. Following, we take the KarushKuhn-Tucker (KKT) optimality conditions. Starting with the stationarity condition we have

$$
\nabla_{r_{i}} L(\mathbf{r}, \mu)=\frac{\rho_{i}}{r_{i}}-\mu_{0}+\mu_{i}=0
$$

since $\rho_{i}>0$, then $\mu_{0}>\mu_{i}$, which also means $\mu_{0}>0$. From the complementary slackness conditions we have

$$
\begin{gathered}
\mu_{0}\left(R^{W i F i}-\sum_{i=1}^{N} r_{i}\right)=0 \\
\mu_{i} r_{i}=0 \\
\mu_{0} \geqslant 0 \text { and } \mu_{i} \geqslant 0, i=1, \ldots, N
\end{gathered}
$$

and since $\mu_{0}>0$, we know that

$$
\sum_{i=1}^{N} r_{i}=R^{W i F i}
$$


which means that $r_{i}, i=1, \ldots, N$ cannot be zero. Thus, forcing $\mu_{i}=0$, $\forall i=1, \ldots N$ we have from $(10)$

$$
r_{i}=\frac{\rho_{i}}{\mu_{0}}
$$

Combining (14) and (15) we have the optimal solution which represents the weighted proportionally fair solution

$$
r_{i}=\frac{\rho_{i}}{\sum_{i=1}^{N} \rho_{i}} R^{W i F i}
$$

Following, we provide an access method that allocates exclusive access to each UE following the PFB algorithm.

\subsection{Implementation Consideration}

In the PFB algorithm we aim to allocate exclusive access periods to each $\mathrm{UE}_{i}$ equal to $t_{i}$, for $i=(1, \ldots, N)$. In these periods the UEs will be able to transmit through the WiFi AP with throughput $R^{W i F i}=S(1)$. We transform the proportionally fair bandwidth allocation into proportionally fair airtime allocation by having $r_{i} \Delta T=t_{i} S(1)$. Now, the weighted proportionally fair airtime allocation is equal to

$$
t_{i}=\frac{\rho_{i}}{\sum_{i=1}^{N} \rho_{i}} \Delta T
$$

Regarding the implementation of the PFB algorithm we aim to give exclusive access to the WiFi AP to each $\mathrm{UE}_{i}$ for a period equal to $t_{i}$. To achieve that, we adopt the idea of unsolicited Clear To Send (CTS) frames initiated by the AP that was proposed in [35]. With a CTS frame the AP protects a specific UE to upload its data through WiFi, while all other UEs put their 802.11 network interface cards into sleep mode for a duration equal to the NAV information of the CTS. A timeline example for the WiFi access of the PFB algorithm for two UEs is presented in Fig. 3. We notice that due to non optimally scheduled user access, $\mathrm{UE}_{2}$ is obliged to wait for a long period in comparison to its own access time. Even though during this waiting period $\mathrm{UE}_{2}$ 's WiFi card is in sleep mode, it consumes energy. We can further improve our algorithm by applying the optimal scheduling for one machine and non-preemptive jobs which is a shortest-job-first fashion approach. 


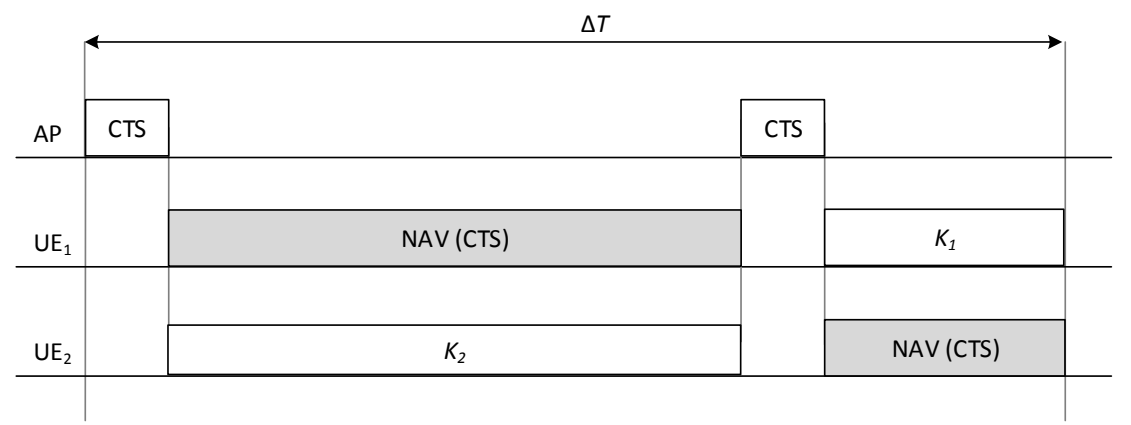

Fig. 3. An example of the PFB algorithm for two UEs.

\subsection{Energy Efficiency of PFB}

The average per UE energy consumption of the WiFi network interface card, during the uploading phase, is expressed as

$$
E C_{T x}^{W i F i}=\frac{1}{N}\left(\sum_{i=1}^{N} \frac{\rho_{i}}{\sum_{i=1}^{N} \rho_{i}} \Delta T \frac{S(1)}{E E(1)}\right) \text { [Joule] }
$$

After scheduling the exclusive time periods $t_{i}$ in augmenting order of duration, the average per UE energy consumption of the WiFi network interface card while in sleep mode with power level $P_{\text {sleep }}^{W i F i}$, is expressed as

$$
E C_{\text {sleep }}^{W i F i}=\frac{1}{N} \sum_{i=1}^{N-1}(N-i) t_{i} P_{\text {sleep }}^{W i F i}[\text { Joule] }
$$

The average per UE energy consumption of the LTE network interface card, due to the concurrent transmission through $\mathrm{WiFi}$, is equal to

$$
E C^{L T E}=\frac{1}{N} \sum_{i=1}^{N}\left(\left(K_{i}-t_{i} S(1)\right) \frac{P_{i}^{L T E}}{\theta_{i} R_{i}^{L T E}}\right) \text { [Joule] }
$$

Combining (18)-(20) the average per UE energy efficiency of IFOM offloading under the PFB algorithm is expressed in (21).

$$
E_{\text {eff }}^{P F B}=\frac{\sum_{i=1}^{N} K_{i}}{N\left(E C_{T x}^{W i F i}+E C_{\text {sleep }}^{W i F i}+E C^{L T E}\right)}[\text { bits/Joule }]
$$




\subsection{Offloading Index}

Aiming to reveal the performance improvement of the PFB algorithm in terms of data volume offloading, we define the WiFi offloading index $O f f_{P F B}$. The $O f f_{P F B}$ is expressed in (22) and is equal to the ratio of the total offloaded data volume through the $\mathrm{WiFi}$ following the $\mathrm{PFB}$ algorithm to the data volume that would be uploaded by the standard 802.11 DCF if only one user was accessing the AP to offload. The WiFi offloading index of the PFB algorithm, $O f f_{P F B}$ is equal to

$$
O f f_{P F B}=\frac{\sum_{i=1}^{N} t_{i} S(1)}{S(1) \Delta T}
$$

It follows from (22) that $O f f_{P F B}=1$, which means that the PFB algorithm fully exploits the offloading capabilities of the WiFi AP, as every UE is allocated exclusive offloading access to the AP.

\section{LTE Pricing Scheme}

The LTE uplink power of each $\mathrm{UE}_{i}$, following the power model of $(1)$, is a function of its LTE uplink transmission rate, $R_{i}^{L T E}$. Hereunder, we propose a two-stage LTE pricing scheme, where the LTE operator decides the price $p$ per unit of uplink transmit rate in the first step and in the second step the UEs decide the rate for which they intend to pay as a function of the price and the spectrum efficiency they experience. We approach the pricing problem using backward induction, examining first the UEs demands (Stage II) and then the operator's decision on the price (Stage I). We propose two pricing models, one linear and one exponential.

\subsection{LTE Uplink Rate With Linear Pricing}

Stage II: The payoff function of the $\mathrm{UE}_{i}$, for acquiring $R_{i}^{L T E}$ quantity of uplink rate with a price $p$ per unit of rate, following the linear pricing model, is expressed as

$$
U_{i}^{l i n}\left(R_{i}^{L T E}\right)=\ln \left(1+\theta_{i} R_{i}^{L T E}\right)-p R_{i}^{L T E}
$$

This payoff function of a $\mathrm{UE}_{i}$, with normalized spectrum efficiency $\theta_{i}$, is equal to the logarithmic utility function, that expresses the diminishing return of getting additional resources, minus the linear price that the $\mathrm{UE}_{i}$ has to pay 
for acquiring $R_{i}^{L T E}$ quantity of rate. We notice that $U_{i}^{l i n}\left(R_{i}^{L T E}\right)$ is a concave function, since $U_{i}^{\text {lin }}\left(R_{i}^{L T E}\right)^{\prime \prime}=-\left(\theta_{i} /\left(1+\theta_{i} R_{i}^{L T E}\right)\right)^{2}<0$. Thus, it has only one maximum, and therefore the local maximum is also the global maximum. Differentiating (23) we have

$$
\frac{\partial U_{i}^{l i n}}{\partial R_{i}^{L T E}}=\frac{\theta_{i}}{1+\theta_{i} R_{i}^{L T E}}-p=0
$$

The optimal value of rate that maximizes $\mathrm{UE}_{i}$ 's payoff is

$$
R_{i}^{* L T E}=\left\{\begin{array}{lr}
\frac{1}{p}-\frac{1}{\theta_{i}}, & \text { if } p \leq \theta_{i} \\
0, & \text { otherwise }
\end{array}\right.
$$

Stage I: We take into consideration that the $N$ UEs that are under the coverage of the same WiFi AP are close enough to present similar channel statistics regarding their LTE connection. Thus, we assume that their spectrum efficiency is such that $\max \left(\theta_{i}\right)-\min \left(\theta_{i}\right)<\varepsilon$, where $\varepsilon>0$. Under this assumption, the operator's choice of price $p$ is such, that the UE with the $\max \left(\theta_{i}\right)$ is allocated the maximum value of the LTE uplink rate $R_{\max }^{L T E}$, aiming to provide the best available service to UEs with better channel conditions compared to the rest of the UEs situated under the same WiFi AP coverage. We also assume that the eNodeB has adequate available resources to satisfy the requests of all UEs. The price is formed according to (26).

$$
p=\frac{\max \left(\theta_{i}\right)}{1+\max \left(\theta_{i}\right) R_{\max }^{L T E}}
$$

The provider aims to give to every $\mathrm{UE}_{i}$ the opportunity to transmit through the LTE. This means that even for the UE with the $\min \left(\theta_{i}\right)$, the quantity $1 / p-1 / \min \left(\theta_{i}\right)$ is positive. Using (26) we find the range of values of $\varepsilon$ under which this rate allocation is feasible. This range is expressed as

$$
0<\varepsilon \leq \max \left(\theta_{i}\right) \min \left(\theta_{i}\right) R_{\max }^{L T E}
$$

The allocated rate to each $\mathrm{UE}_{i}$ following the linear pricing model is expressed as

$$
R_{i}^{L T E}=\frac{1+\max \left(\theta_{i}\right) R_{\max }^{L T E}}{\max \left(\theta_{i}\right)}-\frac{1}{\theta_{i}}
$$




\subsection{LTE Uplink Rate With Exponential Pricing}

For the rate allocation with the exponential pricing model, we follow the same steps as described in the linear pricing approach.

Stage II: The payoff function of $\mathrm{UE}_{i}$, for acquiring $R_{i}^{L T E}$ quantity of uplink rate when applying the exponential pricing model is expressed as

$$
U_{i}^{e x p}\left(R_{i}^{L T E}\right)=\ln \left(1+\theta_{i} R_{i}^{L T E}\right)-p_{e}\left(e^{R_{i}^{L T E}}-1\right)
$$

This payoff function of a $\mathrm{UE}_{i}$ under exponential pricing, with normalized spectrum efficiency $\theta_{i}$, is equal to the logarithmic utility function, that expresses the diminishing return of getting additional resources, minus the exponential price that the $\mathrm{UE}_{i}$ has to pay for acquiring $R_{i}^{L T E}$ quantity of rate. We notice that $U_{i}^{\exp }\left(R_{i}^{L T E}\right)$ is a concave function, since $U_{i}^{\exp }\left(R_{i}^{L T E}\right)^{\prime \prime}=$ $-\left(\theta_{i} /\left(1+\theta_{i} R_{i}^{L T E}\right)\right)^{2}-p_{e} e^{R_{i}^{L T E}}<0$. Thus, it has only one maximum, and therefore the local maximum is also the global maximum. Differentiating (29) we have

$$
\frac{\partial U_{i}^{e x p}}{\partial R_{i}^{L T E}}=\frac{\theta_{i}}{1+\theta_{i} R_{i}^{L T E}}-p_{e} e^{R_{i}^{L T E}}=0
$$

We need to solve this non-linear equation with respect to $R_{i}^{L T E}$. (30) can be rewritten as

$$
\ln \left(\frac{1}{p_{e}}\right)+\frac{1}{\theta_{i}}=\left(R_{i}^{L T E}+\frac{1}{\theta_{i}}\right)+\ln \left(R_{i}^{L T E}+\frac{1}{\theta_{i}}\right)
$$

For $x=R_{i}^{L T E}+\frac{1}{\theta_{i}}$ and $y=\ln \left(\frac{1}{p_{e}}\right)+\frac{1}{\theta_{i}},(31)$ can be written as

$$
y=x+\ln x
$$

which after some straight forward mathematical manipulations can be written as

$$
x e^{x}=e^{y}
$$

Taking the value of the Lambert $W$ function [36] of each part of (33) and using the Lambert $\mathrm{W}$ function identity $W\left(x e^{x}\right)=x$ we have $x=W\left(e^{y}\right)$. Replacing $x$ and $y$ we have

$$
R_{i}^{L T E}=W\left(\frac{e^{\frac{1}{\theta_{i}}}}{p_{e}}\right)-\frac{1}{\theta_{i}}
$$


Stage I: The price $p_{e}$ that the provider decides in the exponential pricing model is such, that the UE with the $\max \left(\theta_{i}\right)$ is allocated the maximum value of the LTE uplink rate $R_{\max }^{L T E}$. The price is formed according to (35).

$$
p_{e}=\frac{\max \left(\theta_{i}\right)}{\left(1+\max \left(\theta_{i}\right) R_{\max }^{L T E}\right) e^{R_{\max }^{L T E}}}
$$

As the provider aims to give to all $N$ UEs under the coverage of the AP the opportunity to upload part of their data needs through the eNodeB, the rate that will be allocated to the user with the $\min \left(\theta_{i}\right)$ should be positive. This means that the range of the spectrum efficiency of the $N$ UEs is such, that

$$
W\left(\frac{e^{\frac{1}{\min \left(\theta_{i}\right)}}}{p_{e}}\right)-\frac{1}{\min \left(\theta_{i}\right)}>0
$$

The allocated rate to each $\mathrm{UE}_{i}$ following the exponential pricing model is expressed as

$$
R_{i}^{L T E}=W\left(\frac{\left(1+\max \left(\theta_{i}\right) R_{\max }^{L T E}\right) e^{R_{\max }^{L T E}+\frac{1}{\theta_{i}}}}{\max \left(\theta_{i}\right)}\right)-\frac{1}{\theta_{i}}
$$

\section{Evaluation and Simulation Results}

We evaluate our offloading schemes by running extensive simulations using MATLAB ${ }^{\mathrm{TM}}$. We run the PFB algorithm for a diverse number of UEs under the concurrent coverage of an eNodeB and a WiFi AP, namely for four to 20 UEs. During the examined offloading periods, each $\mathrm{UE}_{i}$ under the concurrent coverage of the same eNodeB and WiFi AP has $K_{i}$ data volume needs. Hence, every UE has frames to transmit in its buffer and the traffic is considered under saturation conditions. By following this assumption, we provide results for the worst case traffic scenario. We compare the performance of PFB in terms of energy efficiency and offloading capabilities with the standard 802.11 DCF and with an access mechanism titled Smart Exponential-Threshold-Linear (SETL) that was proposed in [37]. In the backoff algorithm proposed in SETL the Contention Window $(C W)$ of a 802.11 user is increasing exponentially up to a threshold that is equal to $C W_{\text {th }}=\left(C W_{\max } / 2+C W_{\min }\right)$. After this threshold, it is increasing linearly up to $C W_{\max }$ according to $C W_{t h}+k C W_{\min }$, where $k$ is a positive integer. 
Tab. 2. Simulation Parameters

\begin{tabular}{ll}
\hline \hline Parameter & Value \\
\hline LTE uplink rate (max) $R_{\max }^{L T E}$ & $5 \mathrm{Mbps}$ \\
LTE uplink power per Mbps $\alpha_{u}$ & $438.39 \mathrm{~mW} / \mathrm{Mbps}$ \\
LTE base power $\beta$ & $1288.04 \mathrm{~mW}$ \\
LTE uplink power & $\alpha_{u} R_{L T E}^{u l}+\beta \mathrm{mW}$ \\
WiFi packet payload & $1500 \mathrm{bytes}$ \\
WiFi Data/ Ctrl. transmission rate & $54 / 6 \mathrm{Mbps}$ \\
WiFi Tx/ Rx/ Idle/ Sleep Power & $1900 / 1340 / 1340 / 75 \mathrm{~mW}$ \\
SIFS/ DIFS & $10 / 50 \mu \mathrm{sec}$ \\
Offloading period $\Delta T$ & $5 \mathrm{sec}$ \\
Number of UEs & $4-20$ \\
Uplink data volume per UE & $5-15 \mathrm{MB}$ \\
\hline \hline
\end{tabular}

Regarding the LTE part of IFOM, we conduct the simulations applying the presented pricing models, i.e. the linear and the exponential pricing approaches. In more detail, the WiFi AP is aware of the upload data needs of each $\mathrm{UE}_{i}$. Based on these data needs and the LTE spectrum efficiency of each $\mathrm{UE}_{i}$, it allocates access time for offloading. The remaining data of each $\mathrm{UE}_{i}$ are uploaded in parallel through its LTE connection with an uplink rate equal to $\theta_{i} R_{i}^{L T E}$, where $R_{i}^{L T E}$ is defined by the applied pricing scheme.

The simulations are repetitively conducted for an offloading time period equal to $\Delta T=5 \mathrm{sec}$. The data volume needs of the UEs are assumed to follow a uniform distribution of the file sizes between $5-15 \mathrm{MB}$. These data needs represent the volume of a photo to a small video, created by contemporary smartphones. The simulations are performed for different value ranges of the spectrum efficiency $\theta_{i}$, aiming to explore the performance of our offloading scheme for offloading regions situated in different distances from the eNodeB. The uplink power level of $\mathrm{UE}_{i}$ 's LTE interface card, $P_{i}^{L T E}$, is assumed to follow (1). The 802.11 network interface card power levels $P_{T x}^{W i F i}, P_{R x}^{W i F i}$, $P_{i d l e}^{W i F i}$ and $P_{\text {sleep }}^{W i F i}$ are assumed to follow the measurements provided in [38]. The numerical values of the simulation parameters are presented in Table 2. 


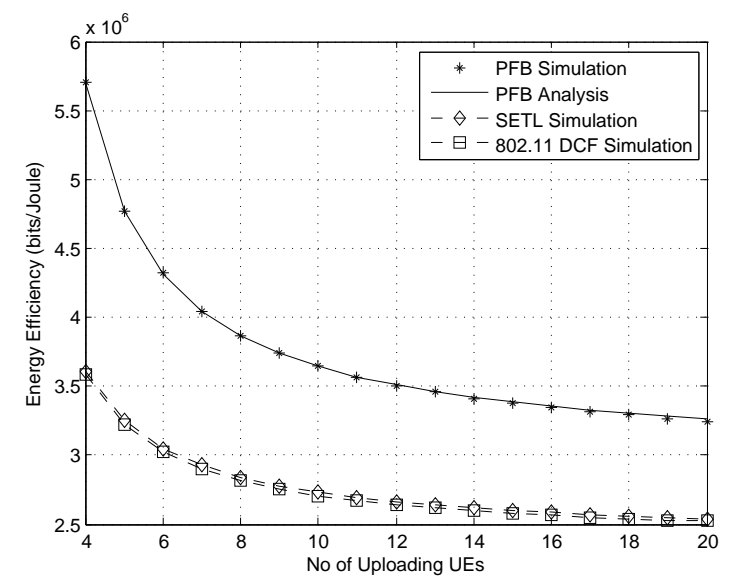

Fig. 4. Energy efficiency for $\theta_{i} \in[0.8,1]$ with linear pricing.

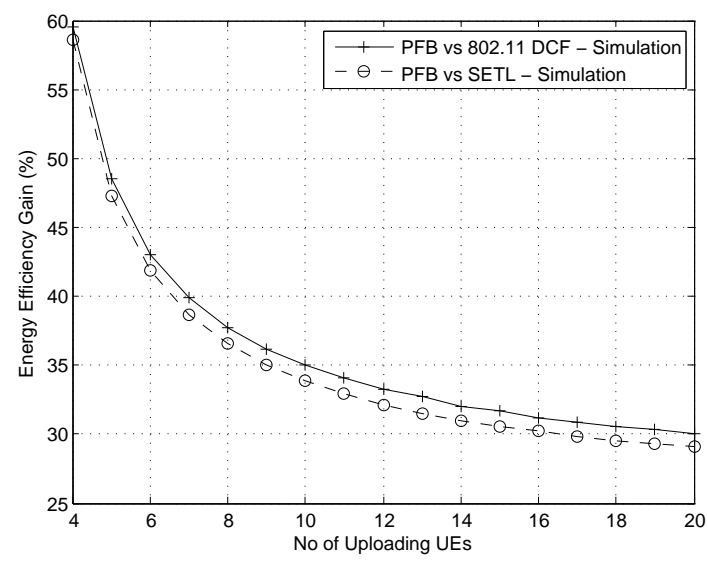

Fig. 5. Energy efficiency gain for $\theta_{i} \in[0.8,1]$ with linear pricing.

\subsection{Energy Efficiency with Linear Pricing}

In Fig. 4 we present the energy efficiency of PFB, 802.11 and SETL, for different number of UEs ranging from four to 20 , with $\theta_{i} \in[0.8,1]$. We notice that as the number of UEs increases the average energy efficiency of a UE decreases because the WiFi bandwidth is shared between more UEs and consequently they have to upload a larger part of their data needs through the energy demanding LTE uplink. The LTE uplink rate allocation follows 


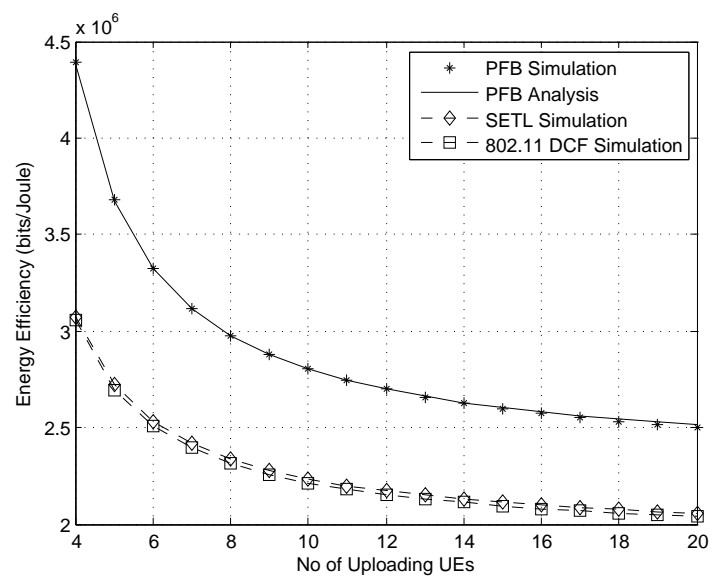

Fig. 6. Energy efficiency for $\theta_{i} \in[0.6,0.8]$ with linear pricing.

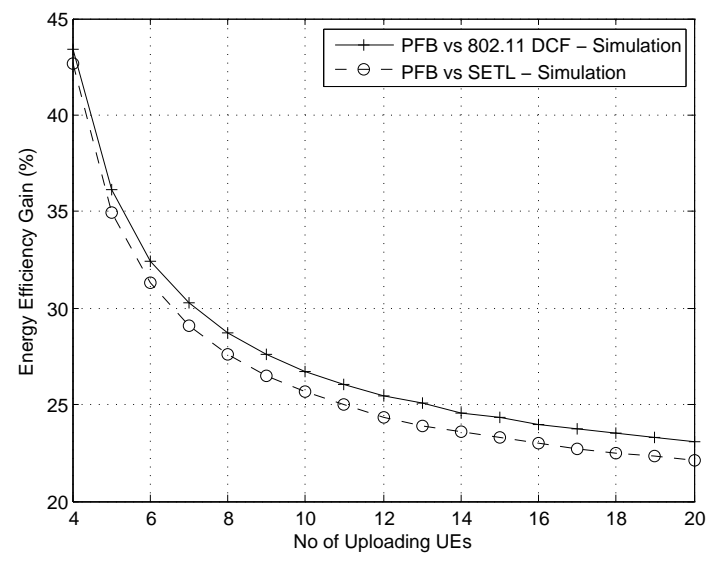

Fig. 7. Energy efficiency gain for $\theta_{i} \in[0.6,0.8]$ with linear pricing.

the linear pricing model. In Fig. 5 the energy efficiency gain (\%) is presented, comparing PFB to the 802.11 standard and the SETL algorithm. For $\theta_{i} \in$ $[0.6,0.8]$ we present the energy efficiency of PFB, 802.11 and SETL in Fig. 6, while in Fig. 7 the energy efficiency gain (\%) is presented, comparing PFB to the 802.11 standard and the SETL algorithm. 


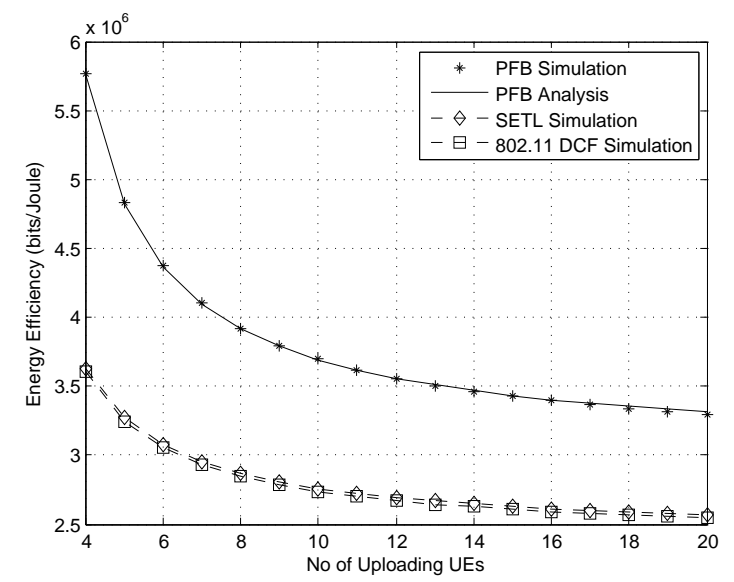

Fig. 8. Energy efficiency for $\theta_{i} \in[0.8,1]$ with exponential pricing.

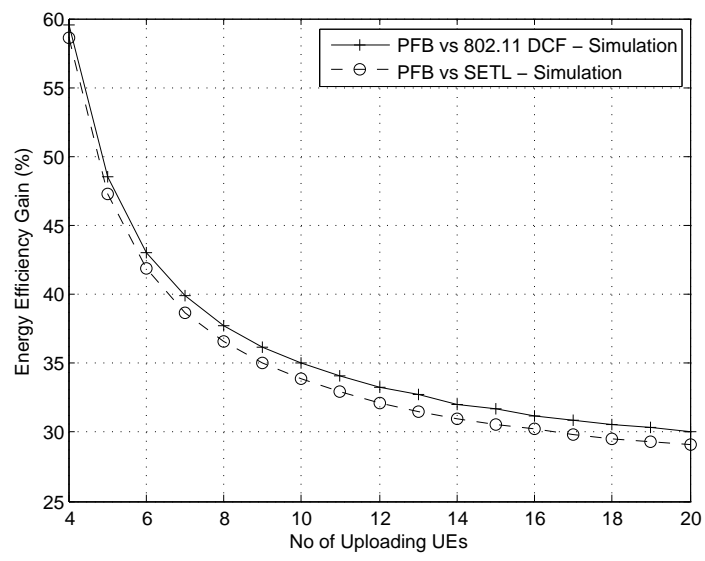

Fig. 9. Energy efficiency gain for $\theta_{i} \in[0.8,1]$ with exponential pricing.

\subsection{Energy Efficiency with Exponential Pricing}

Applying the exponential pricing for the LTE rate allocation we achieve a slight improvement in the energy efficiency of the IFOM offloading scheme. While UEs with lower $\theta_{i}$ are facilitated to acquire more resources, UEs with higher $\theta_{i}$ are pushed to acquire less than they would do by following the linear pricing. In Fig. 8 we present the energy efficiency results following the exponential pricing for $\theta_{i} \in[0.8,1]$, while in Fig. 9 the energy efficiency gain 


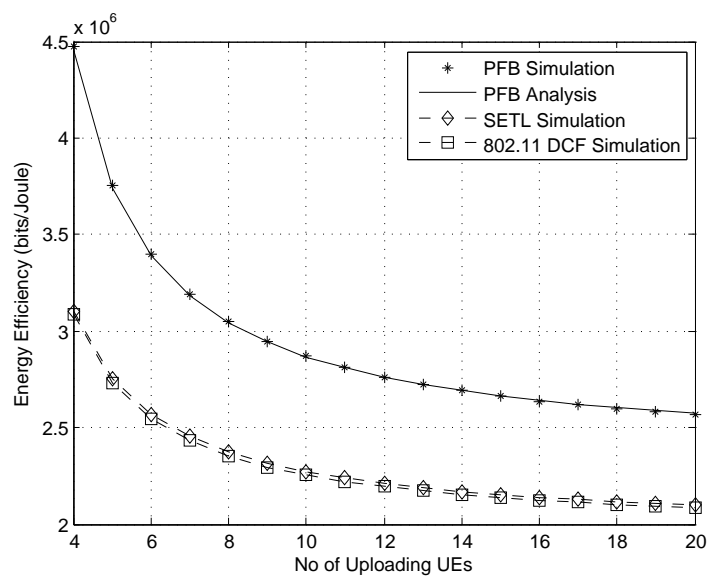

Fig. 10. Energy efficiency for $\theta_{i} \in[0.6,0.8]$ with exponential pricing.

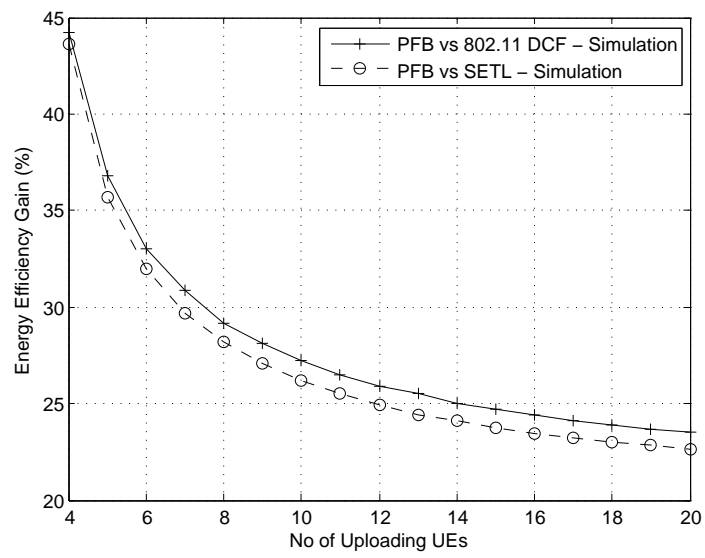

Fig. 11. Energy efficiency gain for $\theta_{i} \in[0.6,0.8]$ with exponential pricing.

is presented for the same range of $\theta_{i}$. In Fig. 10 and 11 we present results for $\theta_{i} \in[0.6,0.8]$.

Comparing the energy efficiency of uplink offloading between linear and exponential pricing schemes, we notice that for $\theta_{i} \in[0.8,1]$ and $\theta_{i} \in[0.6,0.8]$ the performance is similar. Though, for larger range of $\theta_{i}$, e.g. for $\theta_{i} \in[0.2,1]$, and for large number of UEs under the coverage of the same AP, namely from 10 to 20, the exponential pricing scheme presents $20 \%$ better energy 


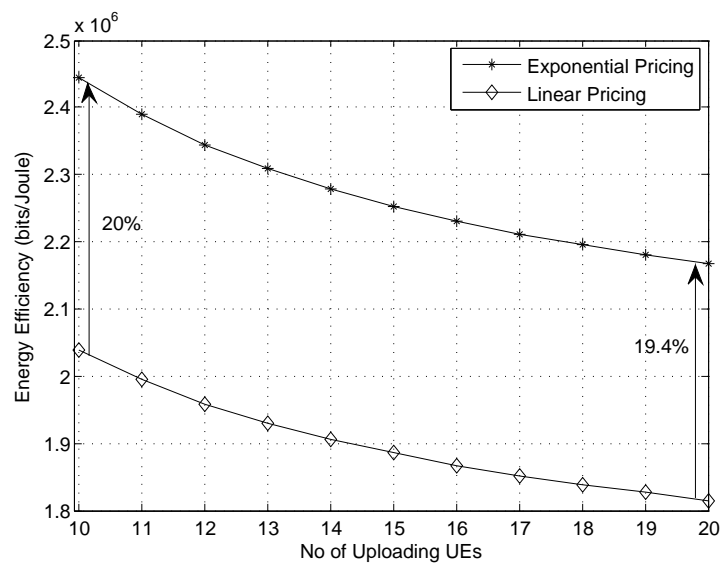

Fig. 12. Energy efficiency comparison between linear and exponential pricing scheme.

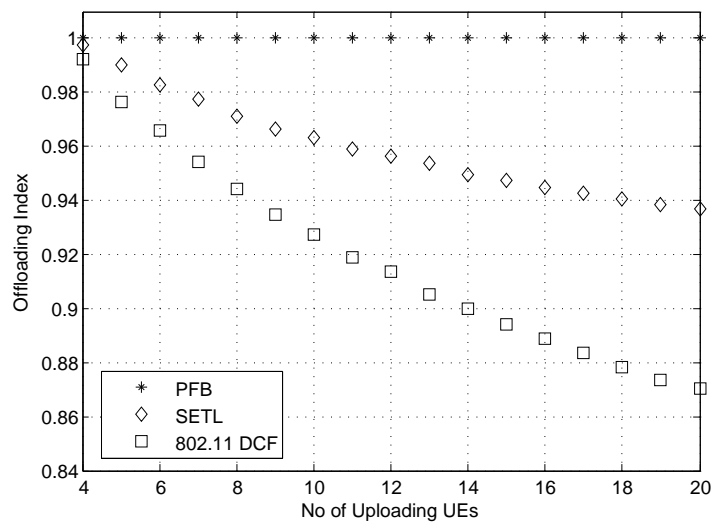

Fig. 13. Offloading Index for different number of UEs.

efficiency compared to the linear pricing scheme. Thus, for greater diversity in the LTE channel quality of the UEs that share the resources of the same AP, the exponential pricing scheme performs better because it gives to UEs that experience worse LTE channel quality the opportunity to purchase more LTE resources, while it pushes UEs with better LTE channel conditions to purchase less resources compared to the linear pricing scheme. Simulation results regarding this comparison are presented in Fig. 12. 


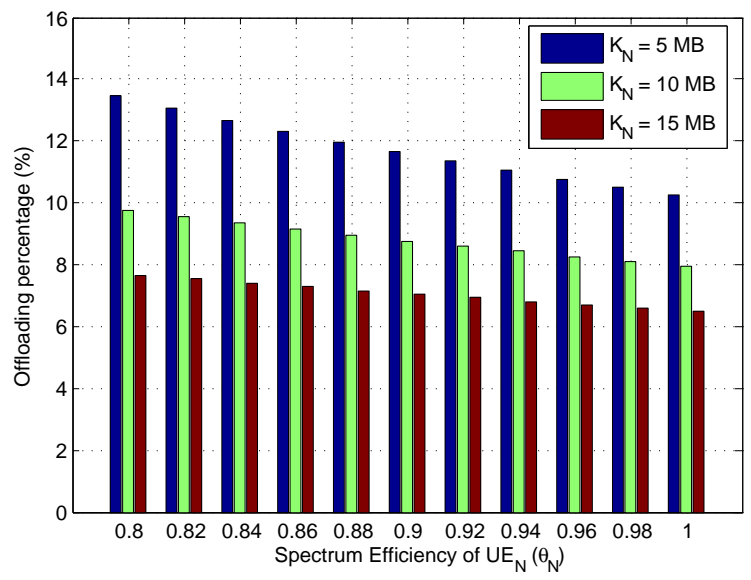

Fig. 14. Impact of the interplay between spectrum efficiency and data volume needs.

\subsection{Evaluation of Offloading Capabilities}

A comparison of the offloading capability of each WiFi uplink approach is presented in Fig. 13. As expected from (22), PFB presents an offloading index equal to one. This means that with $\mathrm{PFB}$ we achieve the maximum exploitation of the APâÁźs capability for offloading. With SETL we achieve an offloading index near 0.94 for high contention conditions (20 offloading UEs) and with 802.11 the offloading index has a value near 0.86 under the same high contention conditions. While PFB provides exclusive WiFi access to the UEs, SETL and 802.11 operate under the CSMA/CA protocol leading to frequent collisions, especially when the number of uploading UEs is increasing.

\subsection{Interplay Between Spectrum Efficiency and Data Volume Needs}

Aiming to reveal the interplay between the spectrum efficiency and the data volume needs of the UEs, we examine our proposed uplink offloading scheme for $N=20$ UEs. We let $\mathrm{UE}_{i}$ for $i \in(1, \ldots, 19)$ with uniformly distributed $\theta_{i} \in[0.8,1]$ and uniformly distributed data needs $K_{i} \in[5,15]$, while we set specific values of $\mathrm{UE}_{N}$ 's spectrum efficiency and data needs. In Fig. 14 we notice that the higher the $\theta_{N}$ the less favoured $\mathrm{UE}_{N}$ is to offload, which is expected as the proportionally fair allocation is weighted by $\rho_{i}=K_{i} / \theta_{i}$. Comparing different data needs $K_{N}$ for the same $\theta_{N}$ we see 


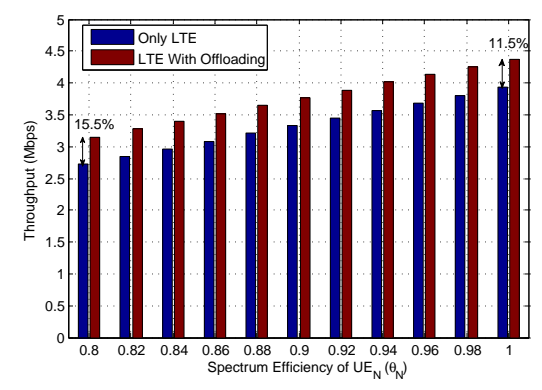

Fig. 15. Throughput comparison for $K_{N}=5 \mathrm{MB}$ and $\theta_{i} \in[0.8,1]$ with linear pricing.

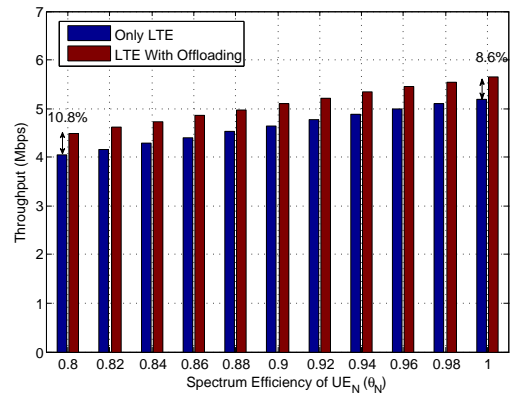

Fig. 16. Throughput comparison for $K_{N}=10 \mathrm{MB}$ and $\theta_{i} \in[0.8,1]$ with linear pricing.

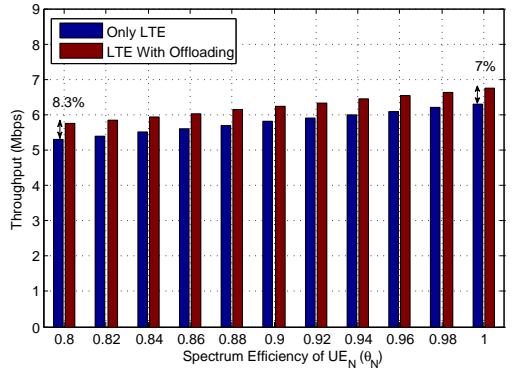

Fig. 17. Throughput comparison for $K_{N}=15 \mathrm{MB}$ and $\theta_{i} \in[0.8,1]$ with linear pricing. 
that the offloading percentage lowers, while the absolute value of the actual offloaded volume raises, which is also expected as $K_{i}$ is in the nominator of the weights $\rho_{i}$. We also compare the aggregate equivalent throughput of $\mathrm{UE}_{N}$ for the concurrent transmission through LTE and WiFi with the case that there was no opportunity to offload and all data were transmitted through LTE, following the linear pricing scheme. Throughput results for $\mathrm{UE}_{N}$ 's data needs $K_{N}=(5,10,15) \mathrm{MB}$, are presented in Fig. 15, 16 and 17 respectively, for $\theta_{i} \in[0.8,1]$, with $i=(1, \ldots, N)$. For these data volume needs and spectrum efficiency range, we achieve a throughput improvement of $7 \%$ to $15.5 \%$, due to the concurrent transmission through LTE and WiFi. Comparing the three figures, we notice that despite the fact that greater $\theta_{N}$ gives less access to offload, the aggregate throughput of $\mathrm{UE}_{N}$ raises due to its improved LTE uplink channel conditions.

\section{Conclusion}

In this paper we focused on the IFOM technique for uplink offloading that allows the concurrent transmission through $\mathrm{WiFi}$ and LTE. UEs are scheduled to upload through the WiFi following a weighted proportionally fair allocation algorithm, where both the data load and the LTE spectrum efficiency is considered in the weighted fairness. Regarding the WiFi access scheme we presented our implementation considerations and proposed a scheme that provides for maximum exploitation of the WiFi resources. For the LTE uplink rate allocation we proposed two pricing algorithms, one linear and one exponential. These algorithms are consisted of two stages. In the first stage the operator chooses the price and in the second stage UEs decide the quantity of resources they intend to acquire based on their payoff functions. We presented an energy efficiency evaluation of our offloading approach for both the linear and the exponential pricing models, comparing at the same time the performance of the proportionally fair access with the standard 802.11 and the SETL algorithm. We also revealed the benefit of exponential pricing in terms of energy efficiency for high diversity of the LTE channel conditions of the UEs and we presented results for the interplay of spectrum efficiency and uplink data needs, and its impact on the offloading percentage of a UE and on its aggregate throughput.

[1] Cisco, Cisco Visual Networking Index: Global Mobile Data Traffic Forecast Update, 2013-âĂŞ2018, Feb. 2014. 
[2] U. Paul, A. P. Subramanian, M. M. Buddhikot, S. R. Das, Understanding Traffic Dynamics in Cellular Data Networks, in: Proc. of IEEE INFOCOM 2011, 2011.

[3] J. Huang, F. Qian, A. Gerber, Z. M. Mao, S. Sen, O. Spatscheck, A Close Examination of performance and Power Characteristics of 4G LTE Networks, in: Proc. of the 10th ACM International Conference on Mobile Systems, Applications, and Services, 2012.

[4] C. Sankaran, Data Offloading Techniques in 3GPP Rel-10 Networks: A Tutorial, IEEE Communications Magazine 50 (6) (2012) 46-53.

[5] ETSI, 3GPP TS 23.261: IP flow mobility and seamless Wireless Local Area Network (WLAN) offload; Stage 2 (v11.0.0), Sep. 2012.

[6] H. Son, S. Lee, S.-C. Kim, Y.-S. Shin, Soft Load Balancing Over Heterogeneous Wireless Networks, IEEE Transactions on Vehicular Technology 57 (4) (2008) 2632-2638.

[7] I. Komnios, F. Tsapeli, S. Gorinsky, Cost-effective multi-mode offloading with peer-assisted communications, Ad Hoc Networks 25 (2015) 370382 .

[8] A. Eryilmaz, R. Srikant, Fair Resource Allocation in Wireless Networks using Queue-length-based Scheduling and Congestion Control, in: Proc. of IEEE INFOCOM 2011, IEEE, 2005.

[9] A. Banchs, P. Serrano, H. Oliver, Proportional Fair Throughput Allocation in Multirate IEEE 802.11e Wireless LANs, Wireless Networks 13 (5) (2007) 649-662.

[10] 802.11e - 2005 - IEEE Standard for Information technology - Local and metropolitan area networks - Specific requirements - Part 11: Wireless LAN Medium Access Control (MAC) and Physical Layer (PHY) Specifications - Amendment 8: Medium Access Control (MAC) Quality of Service Enhancements, IEEE Std 802.11e.

[11] S. Sen, C. Joe-Wong, S. Ha, M. Chiang, Smart Data Pricing (SDP): Economic Solutions to Network Congestion, SIGCOMM eBook on Recent Advances in Networking. 
[12] C. U. Saraydar, N. B. Mandayam, D. Goodman, Efficient Power Control via Pricing in Wireless Data Networks, IEEE Trans. on Communications 50 (2) (2002) 291-303.

[13] P. Liu, P. Zhang, S. Jordan, M. L. Honig, Single-Cell Forward Link Power Allocation Using Pricing in Wireless Networks, IEEE Trans. on Wireless Communications 3 (2) (2004) 533-543.

[14] E.-E. Tsiropoulou, G. K. Katsinis, S. Papavassiliou, Distributed Uplink Power Control in Multiservice Wireless Networks via a Game Theoretic Approach with Convex Pricing, IEEE Trans. on Parallel and Distributed Systems 23 (1) (2012) 61-68.

[15] K. Lee, J. Lee, Y. Yi, I. Rhee, S. Chong, Mobile Data Offloading: How Much Can WiFi Deliver?, IEEE/ACM Trans. on Networking 21 (2) (2013) 536-550.

[16] B. Han, P. Hui, V. Kumar, M. V. Marathe, G. Pei, A. Srinivasan, Cellular Traffic Offloading Through Opportunistic Communications: A Case Study, in: Proc. of the 5th ACM Workshop on Challenged Networks, 2010 .

[17] L. Al-Kanj, H. Poor, Z. Dawy, Optimal Cellular Offloading via Deviceto-Device Communication Networks With Fairness Constraints, IEEE Trans. on Wireless Communications 13 (8) (2014) 4628-4643.

[18] H. Izumikawa, J. Katto, Rocnet: Spatial mobile data offload with userbehavior prediction through delay tolerant networks, in: Wireless Communications and Networking Conference (WCNC), 2013 IEEE, IEEE, 2013, pp. 2196-2201.

[19] A. Balasubramanian, R. Mahajan, A. Venkataramani, Augmenting Mobile $3 \mathrm{G}$ Using WiFi, in: Proc. of the 8th ACM International Conference on Mobile Systems, Applications, and Services, 2010.

[20] G. Iosifidis, L. Gao, J. Huang, L. Tassiulas, An Iterative Double Auction for Mobile Data Offloading, in: Proc of 11th International Symposium on Modeling Optimization in Mobile, Ad Hoc Wireless Networks (WiOpt '13), 2013. 
[21] W. Dong, S. Rallapalli, R. Jana, L. Qiu, K. Ramakrishnan, L. Razoumov, Y. Zhang, T. W. Cho, iDEAL: Incentivized Dynamic Cellular Offloading via Auctions, IEEE/ACM Trans. on Networking 22 (4) (2014) 1271-1284.

[22] M. H. Cheung, J. Huang, Optimal Delayed Wi-Fi Offloading, in: Proc. of 11th IEEE International Symposium on Modeling \& Optimization in Mobile, Ad Hoc \& Wireless Networks (WiOpt '13), 2013.

[23] A. J. Nicholson, B. D. Noble, Breadcrumbs: Forecasting Mobile Connectivity, in: Proc. of the 14th ACM International Conference on Mobile Computing and Networking, 2008.

[24] W. Yoon, B. Jang, Enhanced Non-Seamless Offload for LTE and WLAN Networks, IEEE Communications Letters 17 (10) (2013) 1960-1963.

[25] E. Patouni, N. Alonistioti, L. Merakos, Modeling and Performance Evaluation of Reconfiguration Decision Making in Heterogeneous Radio Network Environments, IEEE Trans. on Vehicular Technology, 59 (4) (2010) 1887-1900.

[26] S.-I. Sou, Mobile Data Offloading With Policy and Charging Control in 3GPP Core Network, IEEE Trans. on Vehicular Technology, 62 (7) (2013) 3481-3486. doi:10.1109/TVT.2013.2255899.

[27] B. H. Jung, N.-O. Song, D. K. Sung, A Network-Assisted User-Centric WiFi-Offloading Model for Maximizing Per-User Throughput in a Heterogeneous Network, IEEE Trans. on Vehicular Technology, 63 (4) (2014) 1940-1945.

[28] J. Parikh, Technical talk in Open Compute Summit (July 2013). URL http://www. oscon.com/oscon2013/public/schedule/detail/29558

[29] V. Miliotis, L. Alonso, C. Verikoukis, Offloading with IFOM: The Uplink Case, in: IEEE Global Communications Conference (GLOBECOM), IEEE, 2014, pp. 2661-2666.

[30] V. Miliotis, L. Alonso, C. Verikoukis, Energy Efficient Proportionally Fair Uplink Offloading for IP Flow Mobility, in: IEEE 19th International Workshop on Computer Aided Modeling and Design of Communication Links and Networks (CAMAD), IEEE, 2014, pp. 6-10. 
[31] IEEE Standard for Information technology - Telecommunications and information exchange between systems - Local and metropolitan area networks - Specific requirements - Part 11: Wireless LAN Medium Access Control (MAC) and Physical Layer (PHY) specifications, IEEE Std 802.11-2007 (Revision of IEEE Std 802.11-2007).

[32] G. Bianchi, Performance Analysis of the ieee 802.11 Distributed Coordination Function, IEEE JSAC 18 (3) (2000) 535-547.

[33] F. P. Kelly, A. K. Maulloo, D. K. Tan, Rate control for communication networks: shadow prices, proportional fairness and stability, Journal of the Operational Research society (1998) 237-252.

[34] S. Boyd, L. Vandenberghe, Convex Optimization, Cambridge University Press, 2009.

[35] Y. Li, D. Papagiannaki, A. Sheth, Uplink Traffic Control in Home 802.11 Wireless Networks, in: Proc. of the 2nd ACM SIGCOMM Workshop on Home Networks, 2011.

[36] R. M. Corless, G. H. Gonnet, D. E. Hare, D. J. Jeffrey, D. E. Knuth, On the Lambert W Function, Advances in Computational Mathematics 5 (1) (1996) 329-359.

[37] C. H. Ke, C. C. Wei, K. W. Lin, J. W. Ding, A Smart ExponentialThreshold-Linear Backoff Mechanism for ieee 802.11 WLANs, International Journal of Communication Systems 24 (8) (2011) 1033-1048.

[38] J. Ebert, S. Aier, G. Kofahl, A. Becker, B. Burns, A. Wolisz, Measurement and Simulation of the Energy Consumption of an WLAN Interface, Technical University of Berlin, Telecommunication Networks Group, Tech. Rep. TKN-02-010. 